\title{
A PROSPECTIVE STUDY ON ROLE OF VITAMIN E SUPPLEMENTATION IN TYPE 2 DIABETES MELLITUS
}

\section{PAVITHRA D ${ }^{1}$, PRAVEEN ${ }^{2}{ }^{2}$, RANADHEER CHOWDARY P $^{2}$, VIJEY AANANDHI M $^{3 *}$}

${ }^{1}$ Department of Pharmacy Practice, School of Pharmaceutical Sciences, Vels Institute of Science, Technology and Advanced Studies, Chennai, Tamil Nadu, India. ${ }^{2}$ Research Scholar, School of Pharmaceutical Sciences, Vels Institute of Science, Technology and Advanced Studies, Chennai, Tamil Nadu, India. ${ }^{3}$ Department of Pharmaceutical Chemistry and Analysis, School of Pharmaceutical Sciences, “Vels Institute of Science, Technology and Advanced Studies, Chennai, Tamil Nadu, India. Email: hodpchemistry@velsuniv.ac.in

Received: 10 October 2018, Revised and Accepted: 11 December 2018

\section{ABSTRACT}

Aim and Objective: The aim of the study was to evaluate the role of Vitamin E supplementation in Type II diabetes mellitus (DM), to determines whether people with Type II DM treated with hypoglycemic agents alone, with or without Vitamin E, to determines the drug interaction in such treatment regimen, and to evaluates the Safety of the regimen.

Methods: Type II DM patients with or without complications were included in this study along with serum glycated hemoglobin (HbA1c) concentration between $7.5 \%$ and $9.5 \%$. They are divided into test group (which received hypoglycemic agent along with Vitamin E 4000 IU) and control group. Body mass index (BMI) status, fasting blood sugar (FBS), and post-prandial blood sugar (PPBS) were noted once in a month, HbA1c percentage, total cholesterol level (TC), and serum Vitamin E level were estimated and noted for every 3 months at total 9 months of this study. Patients with other comorbid conditions were prominent in this study.

Results: It is perceptible with the analysis of obtained data that FBS, PPBS, HbA1c percentage, TC level, and BMI status of the patients were declined gradually in test group (patients with Vitamin E supplementation along with their hypoglycemic agents). Thus, antioxidant therapy is highly propitious whereby delaying the onset of complications in patients with DM. This development would be highly helpful for diabetic patients.

Keywords: Antioxidant, Type II diabetes mellitus, Vitamin E, Complications.

(C) 2018 The Authors. Published by Innovare Academic Sciences Pvt Ltd. This is an open access article under the CC BY license (http://creativecommons. org/licenses/by/4. 0/) DOI: http://dx.doi.org/10.22159/ajpcr.2018.v11s4.31684

\section{INTRODUCTION}

Diabetic may be a cluster of metabolic diseases characterized by symptoms that are hyperbolic blood glucose level ensuring from defects in endocrine secretion, endocrine action, or both [1]. Toxic gas free radicals are involved within the pathological process of diabetes, and its small and macrovascular complications [1]. An imbalance which ends from an inflated production and/or the reduced scavenging of those free radicals ends up in a metabolic state of aerophilous stress that consequently ends up in tissue injury. Motorcar glycosylation reactions alterations within the sorbitol pathway and hyperglycemia are planned as a number of the mechanisms that square measure accountable for this inflated aerophilous stress $[2,3]$. The injury that is finished to the building block by the reactive gas species (oxidative damage) is unbroken under control by a network of inhibitor defense and repair systems that square measure synthesized inside the physique [4,5]. In addition, antioxidants square measure obtained from the diet. One in all the most effective characterized of those is Vitamin E, a vitamin that helps in preventing injury to the lipids by the gas free radicals. When highly-reactive species attack the lipids inside the membranes or the lipoproteins, they depart the chain reaction of supermolecule per chemical reaction [6]. Vitamin E halts this chain reaction, for example, it acts as a sequence breaking matter of supermolecule per chemical reaction. The chronic symptoms of the polygenic disease are related to long harm, dysfunction, and failure of various organs, together with eyes, kidney, nerves, heart, and blood vessels, Vitamin $\mathrm{E}$ has inhibitor activity $[7,8]$. It's going to even have antiatherogenic, antithrombotic, medicinal drug, neuroprotective, antiviral, and immunomodulatory. Vitamin $\mathrm{E}$ may be a collective term used to describe eight separate forms, the best-known form being alpha-tocopherol [9]. Vitamin $\mathrm{E}$ is a fat-soluble vitamin and is an important antioxidant. It acts to safeguard cells against the results of free radicles, that square measure the probably measuring by-products of the body's metabolism [11-13]. Antioxidant such as Vitamin E facilitates defend against the damaging effects of free radicles, which can contribute to the event of chronic disease such as cancer, complications in diabetes mellitus (DM). It additionally protects alternative fat-soluble vitamins (A and cluster vitamins) from destruction y element. Low levels of Vitamin E are joined to the hyperbolic incidence of long-time complication in DM [14-16].

\section{METHODS}

Type II DM patients with or without complications were included in this study along with serum glycated hemoglobin (HbA1c) concentration above $7.5 \%$. They were divided into a test group (which received hypoglycemic agents along with Vitamin E) and the control group. Body mass index (BMI) status, fasting blood sugar (FBS), post-prandial blood sugar (PPBS), HbA1c percentage, total cholesterol (TC) level, and serum Vitamin E level were estimated and noted for every 3 months at total 9 months of this study. Patients with other comorbid conditions were prominent in this study.

\section{Study design}

A prospective study has been carried out with patient's case sheets.

\section{Evaluation test}

Serum glucose and HbA1c are monitored 1 week before treatment initiation, on the day of initiation, $1^{\text {st }}$ and $12^{\text {th }}$ weeks.

\section{Statistical analysis}

Efficacy is analyzed on the basis of Chi-square test with $95 \%$ safety is assessed using student t-test with $95 \%$ 


\section{RESULTS}

Table 1 indicates the gender distribution where male patients were higher in number than female patients. Table 2 shows age distribution, number of patients participating from different age groups. Fig. 1 illustrates the BMI of the patients of different age groups. Fig. 2 illustrates the comorbid conditions of patients participating in this study; hypertension is commonly seen in almost all the patients. Table 3 indicates the base line, average values before treatment. In Fig. 3 , the graph indicates the BMI status of the test (Group B) and control (Group A) groups, where the BMI status of the test group was decreased gradually. Table 4 indicates the FBS, Table 5 with PPBS, Table 6 shows the Hba1c levels of test and control groups, where there was a significant decline in the test groups of all three different blood sugar investigation. Table 7 indicates the TC

Table 1: Gender distribution

\begin{tabular}{lll}
\hline S. No & Gender & Number of patients \\
\hline 1 & Male & 70 \\
2 & Female & 30 \\
\hline
\end{tabular}

Table 2: Age distribution

\begin{tabular}{lll}
\hline S. No & Age group & Number of patients \\
\hline 1 & $18-35$ & 9 \\
2 & $36-50$ & 19 \\
3 & $57-65$ & 40 \\
4 & $>65$ & 32 \\
\hline
\end{tabular}

Table 3: BMI

\begin{tabular}{lll}
\hline S. No & BMI & Number of patients \\
\hline 1 & $<19$ & 2 \\
2 & $19-25$ & 51 \\
3 & $>25$ & 47 \\
\hline
\end{tabular}

BMI: Body mass index

Table 4: Baseline characteristics

\begin{tabular}{lllll}
\hline S. No & Characteristics & Group A & Group B & p value \\
\hline 1 & Male & 36 & 34 & 0.9716 \\
2 & Age (years) & $57.61 \pm 3.8$ & $51.62 \pm 1.96$ & 0.0827 \\
3 & BMI & $24.6 \pm 1.8$ & $23.9 \pm 2.1$ & 0.0721 \\
4 & FBS & $161.6 \pm 13.4$ & $168 \pm 14.6$ & 0.0517 \\
5 & PPBS & $239.6 \pm 18.82$ & $237.6 \pm 16.41$ & 0.0978 \\
6 & HbA1c & $8.3 \pm 0.6$ & $8.7 \pm 0.4$ & 0.0589 \\
7 & Sr. Vitamin E levels & $3.9 \pm 0.8$ & $4.06 \pm 0.7$ & 0.0578 \\
8 & LDL & $137.6 \pm 3.2$ & $134.8 \pm 7.1$ & 0.0921 \\
9 & TC & $214.6 \pm 16.8$ & $227.4 \pm 13.2$ & 0.0582 \\
10 & HDL & $47.6 \pm 3.8$ & $44.9 \pm 2.8$ & 0.0716 \\
\hline
\end{tabular}

All values are Mean \pm SEM. BMI: Body mass index, FBS: Fasting blood sugar, PPBS: Post-prandial blood sugar, HbA1c: Glycated hemoglobin,

LDL: Low-density lipoprotein, TC: Total cholesterol, HDL: High-density lipoprotein, SEM: Standard error of the mean level of patients participating in the test and control groups, where there is a significant decrease in the test group compared to the control group. Table 8 indicates the serum Vitamin E level of both the groups where the patients belonging test group were highly benefited with gradually increase of serum Vitamin E level in their body. Fig. 8 shows that there was no significant Adverse Drug Reaction in this study. It is perceptible with the analysis of obtained data that the FBS, PPBS, HbA1c percentage, TC level, and BMI status of the patients were declined gradually in the test group (patients with Vitamin E supplementation along with their hypoglycemic agents). Thus, the antioxidant therapy is highly propitious whereby delaying the onset of complications in patients with DM. This development would be highly helpful for diabetic patients.

\section{DISCUSSION}

At the initial stage, the history of the patients was collected. General physical examinations and systemic examinations were carried out. The routine blood parameters such as FBS, PPBS, HbA1c and serum Vitamin $\mathrm{E}$ level were estimated. A routine urine examination was done. The patients were followed up once a month, and $\mathrm{HbA1c}$ percentage and serum Vitamin E level were noted for every 3 months at total 9 months of this study. The history regarding the complications was

Table 5: BMI

\begin{tabular}{lll}
\hline S. No & BMI & Number of patients \\
\hline 1 & $<19$ & 2 \\
2 & $19-25$ & 51 \\
3 & $>25$ & 47 \\
\hline BMI: Body mass index & &
\end{tabular}

Table 6: Fasting blood sugar

\begin{tabular}{lllll}
\hline S. No & Groups & Group A & Group B & p value \\
\hline 1 & Pre-treatment & $161.6 \pm 13.4$ & $168.3 \pm 14.6$ & 0.0517 \\
2 & Post-treatment & $149.7 \pm 12.6$ & $139.6 \pm 10.2$ & 0.0497 \\
3 & p value & 0.0521 & 0.0317 & \\
\hline
\end{tabular}

All values are mean \pm SEM. SEM: Standard error of the mean

Table 7: Post-prandial blood sugar

\begin{tabular}{lllll}
\hline S. No & Groups & Group A & Group B & p value \\
\hline 1 & Pre-treatment & $296 \pm 3.64$ & $290 \pm 3.15$ & 0.0921 \\
2 & Post-treatment & $288 \pm 3.21$ & $264 \pm 3.16$ & 0.0495 \\
3 & p value & 0.0317 & 0.0392 & \\
\hline
\end{tabular}

All values are mean \pm SEM. SEM: Standard error of the mean

Table 8: HbA1c

\begin{tabular}{lllll}
\hline S. No & Groups & Group A & Group B & p value \\
\hline 1 & Pre-treatment & $8.3 \pm 0.6$ & $8.7 \pm 0.4$ & 0.0589 \\
2 & Post-treatment & $7.9 \pm 0.7$ & $6.9 \pm 0.32$ & $0.0417^{*}$ \\
3 & p value & 0.0591 & $0.0321^{*}$ & \\
\hline
\end{tabular}

All values are mean \pm SEM. SEM: Standard error of the mean

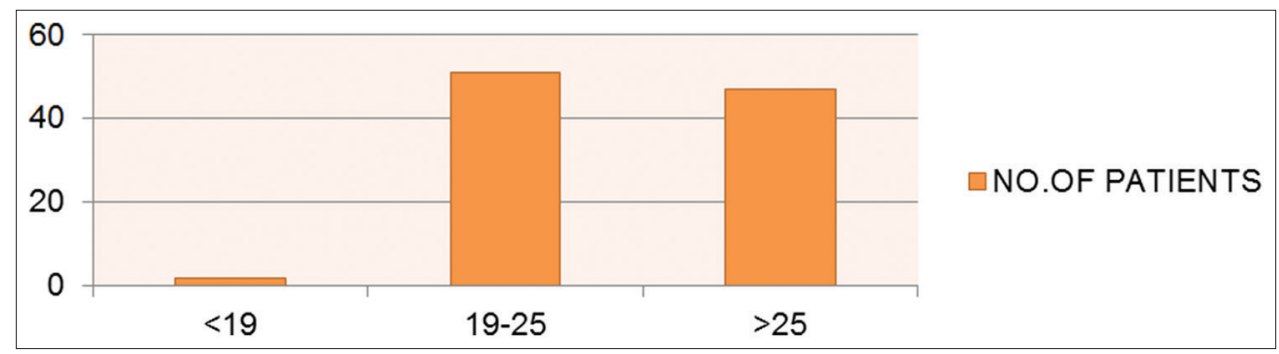

Fig. 1: Body mass index 


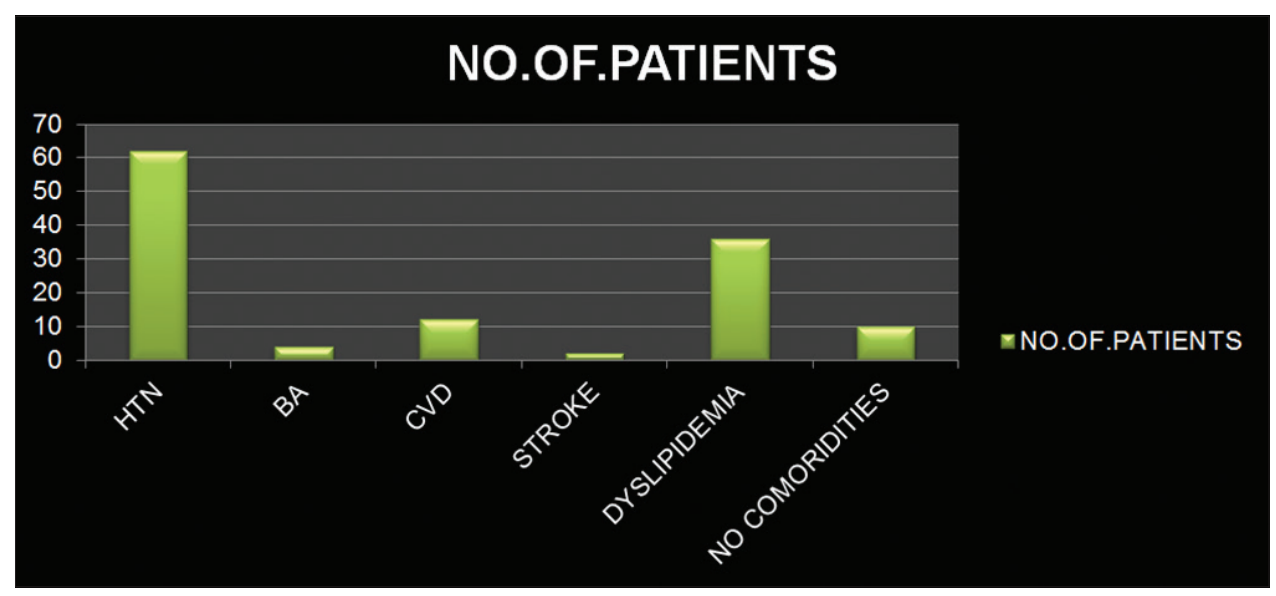

Fig. 2: Comorbid conditions

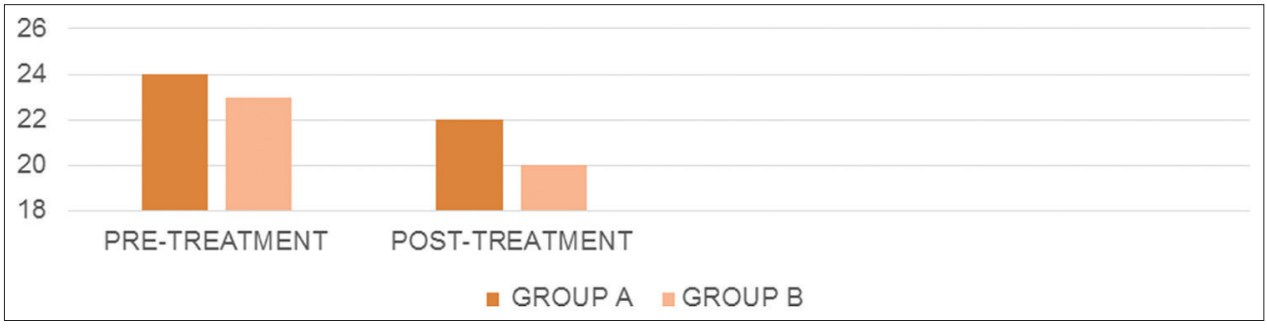

Fig. 3: Body mass index

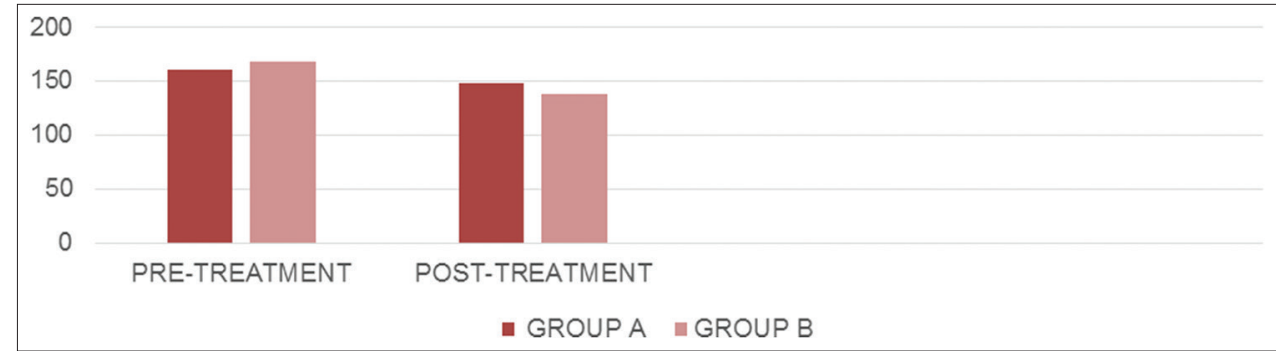

Fig. 4: Fasting blood sugar

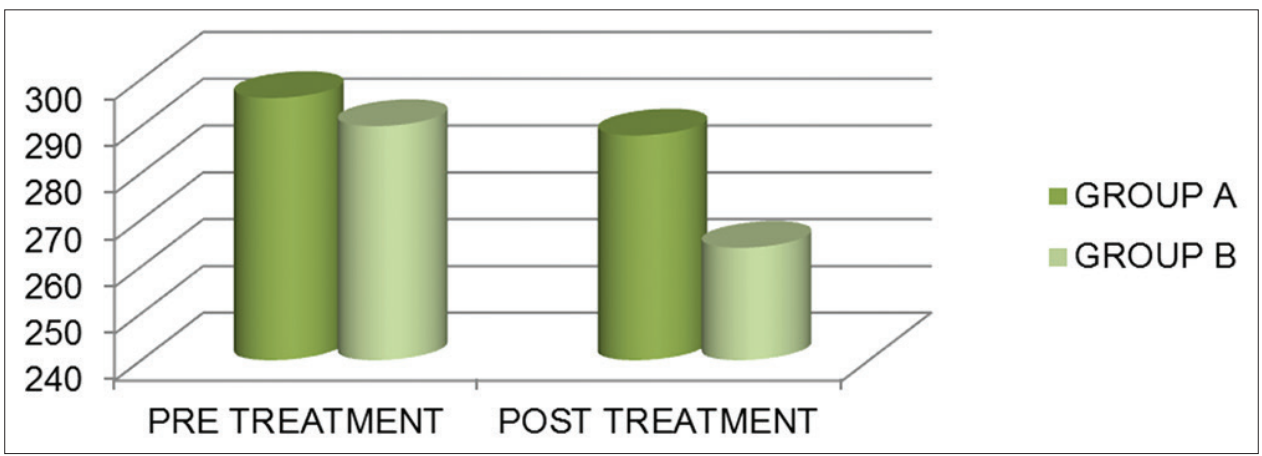

Fig. 5: Post-prandial blood sugar

taken. The routine investigations were done. Fundoscopic examinations were done. The electrocardiography was recorded. Patient counseling regarding medication and diet were given. In Tables 6-8 and Figs 4-7, FBS, PPBS, and Hba1c levels were gradually decreased in test group where the patients in the test group consumed Vitamin E capsule along with their regular hypoglycemic medication, the decline was statistically significant which was similar to the result of Jain et al., in Table 9, there was a significant decline in the TC level in patients of test Group, which was statistically significant which was similar to the result of Kuznetsov et al. and Devaraj and Jialal [10], concluded that the Type II DM patients who were supplemented with tocopherol had a gradual decrease in lipid peroxidation and the free radical production by the circulating monocytes. Table 10 shows a gradual increase in Vitamin E level in patients who have been prescribed with Vitamin E supplementation. Fig. 9 shows the ADR report where there is no significance difference in both the groups. Hence, it also 


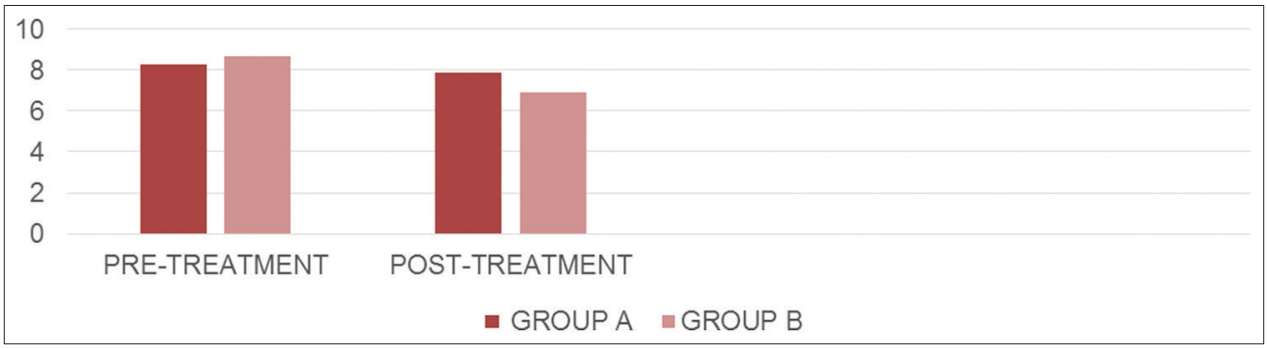

Fig. 6: HbA1c

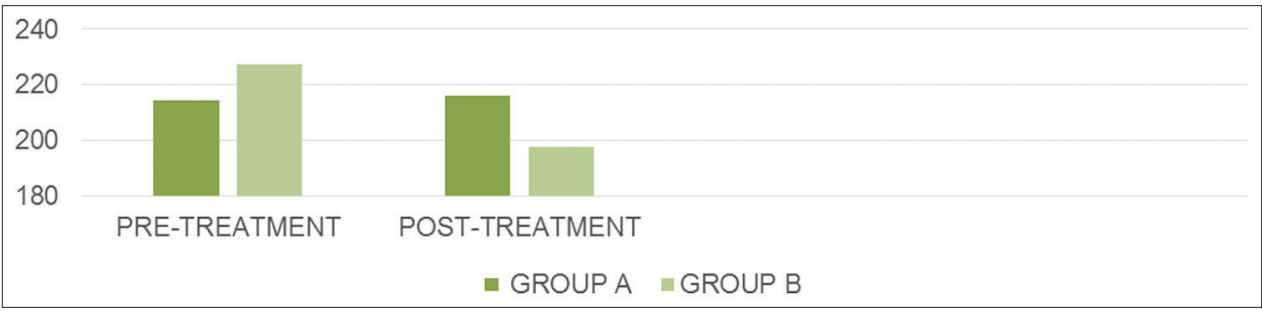

Fig. 7: Total cholesterol

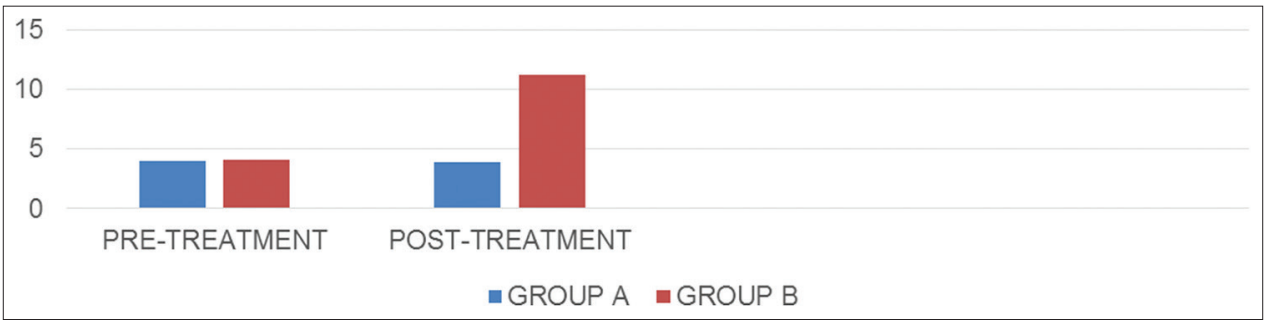

Fig. 8: Serum Vitamin E levels

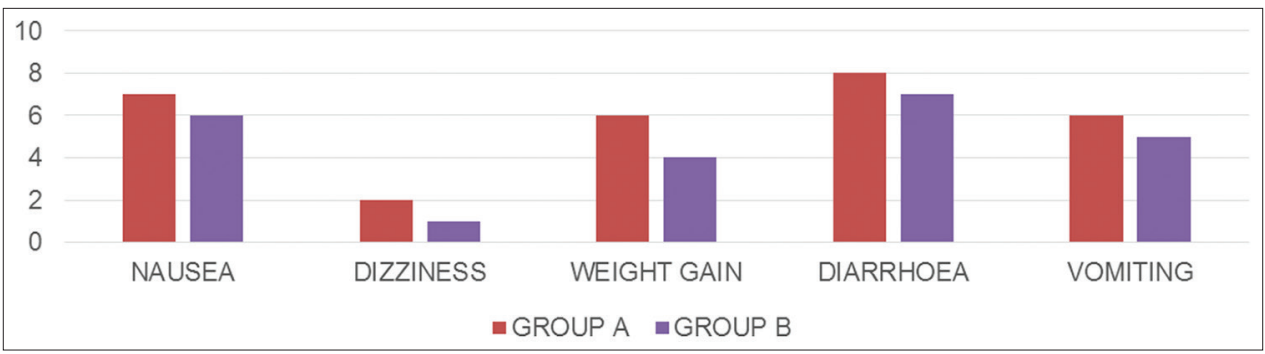

Fig. 9: Adverse drug reaction reported

Table 9: Total cholesterol

\begin{tabular}{lllll}
\hline S. No & Groups & Group A & Group B & p value \\
\hline 1 & Pre-treatment & $214.6 \pm 16.8$ & $227.4 \pm 13.2$ & 0.0582 \\
2 & Post-treatment & $212.2 \pm 13.9$ & $197.6 \pm 6.4$ & 0.0316 \\
3 & p value & 0.9718 & 0.0214 & \\
\hline
\end{tabular}

All values are mean \pm SEM. SEM: Standard error of the mean

Table 10: Serum Vitamin E levels

\begin{tabular}{lllll}
\hline S. No & Groups & Group A & Group B & p value \\
\hline 1 & Pre-treatment & $3.97 \pm 0.8$ & $4.06 \pm 0.7$ & 0.0578 \\
2 & Post-treatment & $3.86 \pm 0.9$ & $11.21 \pm 0.6$ & 0.0027 \\
3 & p value & 0.9916 & 0.0032 & \\
\hline
\end{tabular}

decreased the markers of inflammation, which included the C-reactive protein, interleukin (IL)-1 and IL-6 [17-19]. Fewer studies prove that tocopherols improve the retinal blood flow in patients encountered with diabetic retinopathy complication and thus slowing down the onset of much other complication in Type II DM patients [20,21].

\section{CONCLUSION}

Vitamin E therapy in DM significantly reduces HbA1c and FBS, postprandial in subjects with low and poor glycemic control. Thus, a longterm antioxidant therapy - Vitamin $\mathrm{E}$ is beneficial, as it slows down the onset and slowing down the progression of complications.

\section{ACKNOWLEDGMENT}

The authors are very thankful to Vels Institute of Science Technology and Advanced Studies and its management for providing research facilities and encouragement and also to the patients, who participated in this study.

\section{REFERENCES}

1. Parks E, Traber MG. The mechanisms of the Vitamin E regulation: The research over the past decade and the focus on the future. Antioxid 
Redox Signal 2000;2:405-12

2. Bursell SE, Clermont AC, Aiello LP, Aiello LM, Schlossman DK, Feener EP, et al. High-dose Vitamin E supplementation normalizes retinal blood flow and creatinine clearance in patients with Type 1 diabetes. Diabetes Care 1999;22:1245-51

3. Narang AP, Grewal RK, Kaur S, Gazetteet R. The role of oxidant stress in diabetic retinopathy. Indian Med Gaz 2002;12:1-2.

4. Weiss WP, Hogan JS, Wyatt DJ. Relative bioavailability of all-rac and RRR Vitamin E based on neutrophil function and total alpha-tocopherol and isomer concentrations in periparturient dairy cows and their calves. J Dairy Sci 2009;92:720-31.

5. Sumien N, Forster MJ, Sohal RS. Supplementation with Vitamin E fails to attenuate oxidative damage in aged mice. Exp Gerontol 2003;38:699-704.

6. Bieri JG, Teets L, Belavady B, Andres EL. Serum Vitamin E concentrations in a normal adult population in the Washington, D.C Area. Proc Soc Exp Biol Med 1964;117:131-3.

7. Kuznetsov NS, Abulela AM, Neskoromnyi VN. The use of antioxidants (alpha-tocopherol acetate) in the treatment of diabetes mellitus. Probl Endokrinol (Mosk) 1993;39:9-11.

8. Jain S, Gaiha M, Bhattacharjee J, Auradha S. The effects of a low dose omega 3 fatty acid substituion in Type 2 diabetes mellitus with special reference to the oxidative stress. A prospective preliminary study. J Assoc Physicians India 2002;50:1028-33. Lee IM.

9. Devaraj S, Jialal I. Low-density lipoprotein postsecretory modification, monocyte function, and circulating adhesion molecules in Type 2 diabetic patients with and without macrovascular complications: The effect of alpha-tocopherol supplementation. Circulation 2000;102:191-6

10. Devaraj S, Jialal I. Alpha tocopherol supplementation decreases serum C-reactive protein and monocyte interleukin-6 levels in normal volunteers and Type 2 diabetic patients. Free Radic Biol Med
2000;29:790-2.

11. Upritchard JE, Sutherland WH, Mann JI. Effect of supplementation with tomato juice, Vitamin E, and Vitamin C on LDL oxidation and products of inflammatory activity in Type 2 diabetes. Diabetes Care 2000;23:733-8.

12. Bursell SE, Clermont AC, Aiello LP, Aiello LM, Schlossman DK, Feener EP, et al. High-dose Vitamin E supplementation normalizes retinal blood flow and creatinine clearance in patients with Type 1 diabetes. Diabetes Care 1999;22:1245-51.

13. Zatalia SR, Sanusi H. The role of antioxidants in the pathophysiology, complications, and management of diabetes mellitus. Acta Med Indones 2013;45:141-7.

14. Brun PJ, Yang KJ, Lee SA, Yuen JJ, Blaner WS. Retinoids: Potent regulators of metabolism. Biofactors 2013;39:151-63.

15. Raghow R. Metabolic balancing acts of Vitamin A in Type-2 diabetes and obesity. World J Diabetes 2012;3:174-7.

16. Polidori MC, Mecocci P, Stahl W, Parente B, Cecchetti R, Cherubini A, et al. Plasma levels of lipophilic antioxidants in very old patients with Type 2 diabetes. Diabetes Metab Res Rev 2000;16:15-9.

17. Abahusain MA, Wright J, Dickerson JW, de Vol EB. Retinol, alphatocopherol and carotenoids in diabetes. Eur J Clin Nutr 1999;53:630-5.

18. Reunanen A, Knekt P, Aaran RK, Aromaa A. Serum antioxidants and risk of non-insulin dependent diabetes mellitus. Eur J Clin Nutr 1998;52:89-93.

19. Gavrilov V, Harman-Boehm I, Amichay D, Tessler G, Shuster T, Friger M, et al. Kidney function and retinol status in Type 2 diabetes mellitus patients. Acta Diabetol 2012;49:137-43.

20. Kotnik P, Fischer-Posovszky P, Wabitsch M. RBP4: A controversial adipokine. Eur J Endocrinol 2011;165:703-11.

21. Pullakhandam R, Palika R, Ghosh S, Reddy GB. Contrasting effects of Type 2 and Type 1 diabetes on plasma RBP4 levels: The significance of transthyretin. IUBMB Life 2012;64:975-82. 